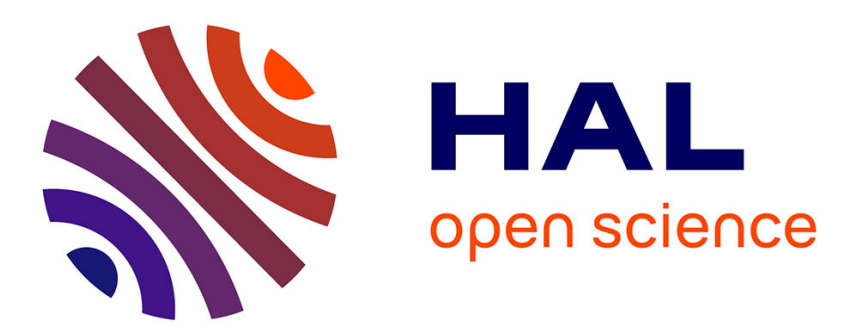

\title{
Ensemble controllability and discrimination of perturbed bilinear control systems on connected, simple, compact Lie groups
}

\author{
Mohamed Belhadj, Julien Salomon, Gabriel Turinici
}

\section{- To cite this version:}

Mohamed Belhadj, Julien Salomon, Gabriel Turinici. Ensemble controllability and discrimination of perturbed bilinear control systems on connected, simple, compact Lie groups. European Journal of Control, 2015, 22, pp.23-29. 10.1016/j.ejcon.2014.12.003 . hal-00866229v4

HAL Id: hal-00866229

https://hal.science/hal-00866229v4

Submitted on 11 Dec 2014

HAL is a multi-disciplinary open access archive for the deposit and dissemination of scientific research documents, whether they are published or not. The documents may come from teaching and research institutions in France or abroad, or from public or private research centers.
L'archive ouverte pluridisciplinaire HAL, est destinée au dépôt et à la diffusion de documents scientifiques de niveau recherche, publiés ou non, émanant des établissements d'enseignement et de recherche français ou étrangers, des laboratoires publics ou privés. 


\title{
Ensemble controllability and discrimination of perturbed bilinear control systems on connected, simple, compact Lie groups
}

\author{
M. Belhadj ${ }^{\mathrm{a}, *}$, J. Salomon ${ }^{\mathrm{b}}$, G. Turinici ${ }^{\mathrm{b}, \mathrm{c}}$ \\ ${ }^{a}$ Département de Mathématiques, Institut Supérieur des Mathématiques Appliquées et d'Informatique de Kairouan, Av. Assad Ibn El Fourat, 3100 \\ Kairouan, TUNISIE \\ ${ }^{b}$ CEREMADE, Université Paris Dauphine, Pl. du Maréchal De Lattre De Tassigny, 75775 Paris Cedex 16, FRANCE \\ ${ }^{c}$ Institut Universitaire de France
}

\begin{abstract}
The controllability of bilinear systems is well understood for finite dimensional isolated systems where the control can be implemented exactly. However when perturbations are present some interesting theoretical questions are raised. We consider in this paper a control system whose control cannot be implemented exactly but is shifted by a time independent constant in a discrete list of possibilities. We prove under general hypothesis that the collection of possible systems (one for each possible perturbation) is simultaneously controllable with a common control. The result is extended to the situations where the perturbations are constant over a common, long enough, time frame. We apply the result to the controllability of quantum systems. Furthermore, some examples and a convergence result are presented for the situation where an infinite number of perturbations occur. In addition, the techniques invoked in the proof allow to obtain generic necessary and sufficient conditions for ensemble controllability.
\end{abstract}

Keywords:

quantum control, Lie group controllability, bilinear system, perturbations

\section{Introduction}

The fundamental importance of addressing the controllability of bilinear systems has long been recognized in engineering control applications (see [II-Q] ]). Among recent applications one may cite the field of quantum control with optical or magnetic external fields (see [5, 9-19]).

Although the controllability is well understood when the system is of finite dimension, isolated and the control can be implemented exactly, new theoretical and numerical questions are raised when perturbations are present.

The question that is addressed in this paper is related to the simultaneous controllability of bilinear systems. Consider a collection of control systems with states $X_{k}$, $k=1, \ldots, K$ in Lie groups $G_{k}$ evolving according to $\frac{d X_{k}(t)}{d t}=\left(A_{k}+u(t) B_{k}\right) X_{k}$. Simultaneous controllability (also called "ensemble controllability") is the ques-

\footnotetext{
${ }^{*}$ Corresponding author. Tel.: +216 77226575

Email addresses: Mohamed.Belhadj@ismai.rnu.tn (M. Belhadj), salomon@ceremade.dauphine.fr (J. Salomon), gabriel.turinici@dauphine.fr (G. Turinici)
}

tion of whether all states $X_{k}$ can be controlled with the same control $u(t)$. We will use the terms "simultaneous controllability" and "ensemble controllability" interchangeably.

Problems of simultaneous control of a finite collection of systems have been addressed recently in applications related to quantum control [20-317]. In such circumstances, the system is a collection of molecules or atoms or spin systems and the control is a magnetic field (in NMR) or a laser. The assessment of whether a single control pulse can drive independent (i.e., distinct) quantum systems to their respective target states was addressed theoretically in [20] for general $A_{k}, B_{k}$ and applied to the optimal dynamic discrimination of separate quantum systems in [21]. The particular case of identical molecules with $A_{k}=A$ (constant) and $B_{k}=\xi_{k} B, \xi_{k} \in$ $\mathbb{R}, G=S U(N)$ was treated in [22, 23] where, under some technical assumptions on $A$ and $B$, it is proved that all members of an ensemble of randomly oriented molecules subjected to a single ultra-fast laser control pulse can be simultaneously controlled. An independent work [30] treats the circumstance when $A_{k}=\epsilon_{k} A$, $\left|\epsilon_{j}\right| \neq\left|\epsilon_{\ell}\right|$ for any $j \neq \ell, G=S U(N)$ and $B_{k}=B$ (con- 
stant) and was used to show controllability for ensembles $N$-level quantum systems having different Larmor dispersion. This last result generalizes the findings of [25] for ensembles of spin $1 / 2$ systems.

The infinite dimensional version (an infinite number of systems $A_{\epsilon}=\epsilon A$ with $\epsilon$ taking arbitrary values in an interval $] \epsilon_{*}, \epsilon^{*}[$ ) was treated in [26, 27, 32] for the specific situation of the Bloch equations.

In this paper, we extend the result in [30] to the new circumstance when $A_{k}=A+\alpha_{k} B, \alpha_{k} \in \mathbb{R}$ and $B_{k}=B$ (constant) or, equivalently, to the simultaneous controllability of systems submitted to time independent perturbations $\frac{d X_{k}(t)}{d t}=\left[A+\left(u(t)+\alpha_{k}\right) B\right] X_{k}$. As the result in [30] does not apply to this situation, we prove new controllability results. Moreover, the mathematical techniques employed in this work turn out to be useful in other settings such as [22, 30] for which we give stronger controllability results.

The perturbation model $A+\left(u(t)+\alpha_{k}\right) B$ was investigated theoretically and numerically in the physical literature independent of any theoretical controllability results. In the quantum computing literature such perturbations are called "fixed systematic errors" (see Section VI.A. equation (40) of [33]) or simply "systematic control error", see [34] where the authors concluded that mitigating such errors may be possible (although at the expense of longer pulse sequences). We give here a theoretical result to sustain this view. We also refer to [35] where the authors design pulse sequences that are generically robust with respect to errors in the amplitude of the control field. In a related recent work the corresponding noise model is called "low frequency noise" (see section IV. C. of [36]): it is defined as the portion of the (control) amplitude noise that has a correlation time that is long (up to $10^{3}$ times) compared to the timescale of the dynamics and as such it can be treated as constant in time. Additional noise models (additive or multiplicative) are presented in [37] in the general quantum control area.

The balance of the paper is as follows: in Section 1 we introduce the general framework and the main notations and in Section [3 we present our main results including a general ensemble controllability result. In Section 4 , we apply our results to the controllability of quantum systems. The situation of an infinite number of perturbations is discussed in Section $\square$ from the theoretical and numerical point of views. Finally, some conclusions and perspectives of future work are given in Section 6 .

\section{Problem formulation}

Let $G$ be a Lie group. Throughout this paper $G$ is considered to be finite dimensional, connected, compact, simple real Lie group. Its Lie algebra is denoted by $\mathfrak{g}$, the identity element is $I d$ and $A, B \in \mathfrak{g}$ are fixed. Remarkable examples of such Lie groups are (see [38, 39]):

- the special unitary group $S U(N)$ for $N \geq 2$,

- the special orthogonal group $S O(N)$ for $N \neq 4$,

- the compact symplectic group (quaternionic $N \times N$ unitary matrices) $\operatorname{Sp}(N)$ for $N \geq 2$,

- the spin group $\operatorname{Spin}(N)$ for $N \geq 2$.

Consider the following control system on $G$ :

$$
\frac{d X(t)}{d t}=(A+u(t) B) X(t), \quad X(0)=I d
$$

The matrix $X(t)$ evolves in the Lie group $G$.

The controllability of a system on Lie groups such as (II) is a well-studied problem [4-9]. The literature on the subject of bilinear control relies essentially on the following Theorem (originally due to [40]):

Theorem 1. Denote by $\mathbb{L}_{A, B}$ the Lie subalgebra of $\mathrm{g}$ generated by $A$ and $B$. The system (D) on the Lie group $G$ is controllable if and only if $\mathbb{L}_{A, B}=\mathfrak{g}$ or equivalently if $\operatorname{dim}_{\mathbb{R}} \mathbb{L}_{A, B}=\operatorname{dim}_{\mathbb{R}}$ g. Moreover there exists $T_{A, B}>0$ such that any target can be reached in time $t \geq T_{A, B}$ with controls $u$ such that $|u(s)| \leq 1, \forall s \in[0, t]$.

Here $\operatorname{dim}_{\mathbb{R}} \mathbb{L}_{A, B}$ stands for the dimension of $\mathbb{L}_{A, B}$ as linear vector space over $\mathbb{R}$.

An important question is what happens if the control $u(t)$ in (UI) is submitted to some perturbations in a predefined (discrete) list $\left\{\alpha_{k}, k=1, \cdots, K\right\}$ ?

$$
\frac{d X_{k}(t)}{d t}=A X_{k}(t)+\left[u(t)+\alpha_{k}\right] B X_{k}(t), X_{k}(0)=I d .
$$

Can one still control the systems simultaneously? The real perturbation $\alpha_{k}$ for a given system is not known beforehand, therefore in order to be certain that the system is controlled, one has to find a control $u(t)$ that simultaneously controls all states $X_{k}(t)$, i.e., find $u(t)$ such that $X_{k}(T)=V$ for $k=1, \cdots, K$ (here $V$ is the target state).

Yet a distinct circumstance is when $\alpha_{k}$ are not arbitrary perturbations but unknown characteristics of the system to be identified. Here, the goal is to find $u(t)$ such that, given distinct $V_{k}$ one has $X_{k}(T)=V_{k}$. By measuring the state of the system at the final time $T$, one knows which $\alpha_{k}$ was effective during $[0, T]$. 
In conclusion, our problem can be formalized as follows: let $V_{k} \in G, k=1, \cdots, K$ be arbitrary. Is it possible to find $T>0$ and a measurable $u:[0, T] \rightarrow \mathbb{R}$ such that the system given by (D) satisfies $X_{k}(T)=V_{k}, \forall k=$ $1, \cdots, K$ ? If the answer to this question is positive then the system in (ㅁ) will be called simultaneously controllable (or ensemble controllable).

\section{Simultaneous controllability for perturbations}

\subsection{Tools for simultaneous controllability}

In this section, we recall an important result on simultaneous controllability. Consider $K$ bilinear systems on the (finite dimensional, connected, compact, simple) Lie groups $G_{k}$ :

$$
\frac{d X_{k}(t)}{d t}=\left(A_{k}+u(t) B_{k}\right) X_{k}(t), X_{k}(0)=I d,
$$

where $A_{k}, B_{k} \in \mathfrak{g}_{k}, k=1, \cdots, K$ and $\mathfrak{g}_{k}$ is the Lie algebra of $G_{k}$. Recall that when $G_{k}$ is simple the Lie algebra $\mathfrak{g}_{k}$ is also simple which means that the only ideals in $\mathfrak{g}_{k}$ are $\{0\}$ and $\mathfrak{g}_{k}$. In particular $\mathfrak{g}_{k}$ is also semi-simple. Let $\mathcal{A}=A_{1} \oplus \cdots \oplus A_{K} \in \oplus_{k=1}^{K} \mathfrak{g}_{k}$ and $\mathcal{B}=B \oplus \cdots \oplus B \in \oplus_{k=1}^{K} \mathfrak{g}_{k}$. When $\mathfrak{g}_{k}$ are represented as matrix algebras and $M_{k} \in \mathfrak{g}_{k}$ the element $M_{1} \oplus \ldots \oplus M_{K} \in \oplus_{k=1}^{K} \mathfrak{g}_{k}$ is simply the block diagonal matrix $\left(\begin{array}{ccc}M_{1} & & 0 \\ & \ddots & \\ 0 & & M_{K}\end{array}\right)$.

By assembling the $K$ bilinear systems (B]), the evolution of this collection of states can be written as a bilinear system on $\oplus_{k=1}^{K} G_{k}$ :

$$
\frac{d \mathbf{X}(t)}{d t}=\mathcal{A} \mathbf{X}(t)+u(t) \mathcal{B} \mathbf{X}(t), \mathbf{X}(0)=\mathbf{I d} \in \oplus_{k=1}^{K} G_{k}
$$

Denote by $\mathbb{L}_{\mathcal{A}, \mathcal{B}}$ the Lie algebra generated by the matrices $\mathcal{A}$ and $\mathcal{B}$. Then, we have the following result (see [40], [20], Theorems 1 and 2 p. 277 and [21], Section III for an application):

Theorem 2. The collection (B) of $K$ bilinear systems is simultaneously controllable if and only if $\mathbb{L}_{\mathcal{A}, \mathcal{B}}=\oplus_{k=1}^{K} \mathfrak{g}_{k}$ or equivalently

$$
\operatorname{dim}_{\mathbb{R}} \mathbb{L}_{\mathcal{A}, \mathcal{B}}=\sum_{k=1}^{K} \operatorname{dim}_{\mathbb{R}} \mathfrak{g}_{k} .
$$

Moreover, there exists $T_{\mathcal{A}, \mathcal{B}}>0$ such that any collection of targets $\left(V_{k}\right)_{k=1}^{K} \in \oplus_{k=1}^{K} G_{k}$ can be reached in time $t \geq$ $T_{\mathcal{A}, \mathcal{B}}$ with controls $u(t)$ such that $|u(s)| \leq 1, \forall s \in[0, t]$.

\subsection{Main result}

The proof of our main result uses the following lemma.

Lemma 3. Consider the collection (B]) of $K$ bilinear systems as a control system on $\oplus_{k=1}^{K} G_{k}$. Suppose $K>1$ and $\mathbb{L}_{A_{k}, B_{k}}=\mathfrak{g}_{k}$ for any $k=1, \cdots, K$. The system is not ensemble controllable if and only if there exist $k, \ell \in\{1, \ldots, K\}, k \neq \ell$ and an isomorphism $f: \mathfrak{g}_{k} \rightarrow \mathfrak{g}_{\ell}$ such that $f\left(A_{k}\right)=A_{\ell}$ and $f\left(B_{k}\right)=B_{\ell}$.

Proof. If such an isomorphism exists then the dynamics of the $\ell$-th system is completely dependent on the dynamics of the $k$-th system, in fact there will be an isomorphism of Lie groups $F: G_{k} \rightarrow G_{\ell}$ such that $X_{\ell}=F\left(X_{k}\right)$ at any time $t$ and with any control $u(t)$. Therefore the collection of systems is not ensemble controllable.

To prove the direct implication, suppose that the collection of $K$ bilinear systems is not ensemble controllable and let $K^{\prime} \leq K$ be the first integer such that the systems associated with $A_{k}, B_{k}, k=1, \ldots, K^{\prime}$ are not ensemble controllable but any $\left(K^{\prime}-1\right)$-tuple $\left\{i_{1}, \ldots, i_{K^{\prime}-1}\right\} \subset$ $\left\{1, \ldots, K^{\prime}-1\right\}\left(i_{k} \neq i_{\ell}\right.$ for $\left.k \neq \ell\right)$ of systems $A_{i_{k}}, B_{i_{k}}$, $k=1, \ldots, K^{\prime}-1$, is ensemble controllable; by hypothesis $K \geq 2$ since any individual system is controllable. To ease notations we renote $K=K^{\prime}$.

Step 1: Denote $\mathfrak{g}^{0}=\left\{\chi \in \mathfrak{g}_{K} \mid 0 \oplus \cdots \oplus 0 \oplus \chi \in \mathbb{L}_{\mathcal{A}, \mathcal{B}}\right\}$. Since $\mathbb{L}_{\mathcal{A}, \mathcal{B}}$ is a linear space $\mathrm{g}^{0}$ will also be a non-empty linear space. Let $\chi \in \mathfrak{g}^{0}$ and $\psi \in \mathfrak{g}_{K}$. Since $\mathbb{L}_{A_{K}, B_{K}}=\mathfrak{g}_{K}$ there exists at least an element of the form $\psi_{1} \oplus \cdots \oplus$ $\psi_{K-1} \oplus \psi \in \mathbb{L}_{\mathcal{A}, \mathcal{B}}$. Recall that $0 \oplus \cdots \oplus 0 \oplus \chi \in \mathbb{L}_{\mathcal{A}, \mathcal{B}}$ thus $0 \oplus \cdots \oplus 0 \oplus[\chi, \psi]=\left[0 \oplus \cdots \oplus 0 \oplus \chi, \psi_{1} \oplus \cdots \oplus \psi_{K-1} \oplus \psi\right] \epsilon$ $\mathbb{L}_{\mathcal{A}, \mathcal{B}}$ therefore $[\chi, \psi] \in \mathfrak{g}^{0}$. We obtain that $\mathfrak{g}^{0}$ is an ideal of $\mathfrak{g}_{K}$. But $\mathfrak{g}_{K}$ is a simple Lie algebra which implies that the only ideals in $\mathfrak{g}_{K}$ are $\{0\}$ and $\mathfrak{g}_{K}$.

We treat first the alternative $\mathfrak{g}^{0}=\mathfrak{g}_{K}$. Let $\chi_{1} \in$ $\mathfrak{g}_{1}, \cdots, \chi_{K} \in \mathfrak{g}_{K}$ be arbitrary. The $(K-1)$-tuple of systems on $\oplus_{k=1}^{K-1} G_{k}$ is controllable therefore the Lie algebra generated by $A_{1} \oplus \cdots \oplus A_{K-1}$ and $B_{1} \oplus \cdots \oplus B_{K-1}$ is $\oplus_{k=1}^{K-1} \mathfrak{g}_{k}$ thus $\mathbb{L}_{\mathcal{A}, \mathcal{B}}$ contains at least one element of the form $\chi_{1} \oplus \cdots \oplus \chi_{K-1} \oplus \widetilde{\chi_{K}}$ for some $\widetilde{\chi_{K}} \in \mathfrak{g}_{K}$. In addition $\mathfrak{g}^{0}=\mathfrak{g}_{K}$ implies that $\chi_{K}-\widetilde{\chi_{K}} \in \mathfrak{g}^{0}$ therefore $0 \oplus \cdots \oplus 0 \oplus\left(\chi_{K}-\widetilde{\chi_{K}}\right) \in \mathbb{L}_{\mathcal{A}, \mathcal{B}}$. Summing the two we obtain $\chi_{1} \oplus \cdots \oplus \chi_{K} \in \mathbb{L}_{\mathcal{A}, \mathcal{B}}$ therefore $\mathbb{L}_{\mathcal{A}, \mathcal{B}}=\oplus_{k=1}^{K} \mathfrak{g}_{k}$ and we obtain controllability which contradicts the hypothesis. It follows that $\mathfrak{g}^{0}=\{0\}$.

Step 2: For any $\chi_{1} \in \mathfrak{g}_{1}, \cdots, \chi_{K-1} \in \mathfrak{g}_{K-1}$ there exists thus a unique element $\chi_{1} \oplus \cdots \oplus \chi_{K} \in \mathbb{L}_{\mathcal{A}, \mathcal{B}}$. Introduce the mapping $J: \oplus_{k=1}^{K-1} \mathfrak{g}_{k} \rightarrow \mathfrak{g}_{K}$ defined by

$$
J\left(\chi_{1}, \cdots, \chi_{K-1}\right)=\chi_{K} \Longleftrightarrow \chi_{1} \oplus \cdots \oplus \chi_{K} \in \mathbb{L}_{\mathcal{A}, \mathcal{B}}
$$


In particular $J\left(B_{1}, \ldots, B_{K-1}\right)=B_{K}$ and $J\left(A_{1}, \ldots, A_{K-1}\right)=$ $A_{K}$. Elementary computations indicate that $J$ is a morphism of Lie algebras, in particular invariant with respect to commutation and $J(0, \ldots, 0)=0$.

Consider $J_{0}: \mathfrak{g}_{1} \rightarrow \mathfrak{g}_{K}$ defined by $J_{0}\left(\chi_{1}\right)=$ $J\left(\chi_{1}, 0, \ldots, 0\right)$. Take $\chi_{1}$ such that $J_{0}\left(\chi_{1}\right)=0$. Then $J\left(\chi_{1}, 0, \ldots, 0\right)=0$ or, equivalently, $\chi_{1} \oplus 0 \oplus \cdots \oplus 0 \in \mathbb{L}_{\mathcal{A}, \mathcal{B}}$. By a reasoning similar to that in Step 1 we prove that $\left\{\chi_{1} \in \mathfrak{g}_{1} \mid \chi_{1} \oplus 0 \oplus \cdots \oplus 0 \in \mathbb{L}_{\mathcal{A}, \mathcal{B}}\right\}$ must be $\{0\}$ thus $\chi_{1}=0$. Therefore we proved $J_{0}\left(\chi_{1}\right)=0$ implies $\chi_{1}=0$ which means that $J_{0}$ is injective. Since the $(K-1)$-tuple of systems $A_{k}, B_{k}, k=2, \ldots, K$ is ensemble controllable, for any $\chi_{2} \in \mathfrak{g}_{2}, \ldots, \chi_{K} \in \mathfrak{g}_{K}$ the algebra $\mathbb{L}_{\mathcal{A}, \mathcal{B}}$ contains at least one element of the form $\chi_{1} \oplus \chi_{2} \oplus \cdots \oplus \chi_{K}$. Considering $\chi_{2}=0, \ldots \chi_{K-1}=0$ and $\chi_{K}$ arbitrary, we find that for any $\chi_{K} \in \mathfrak{g}_{K}$ at least one $\chi_{1} \in \mathfrak{g}_{1}$ exists such that $\chi_{K}=J\left(\chi_{1}, 0, \ldots, 0\right)=J_{0}\left(\chi_{1}\right)$. Therefore $J_{0}$ is also surjective thus bijective. Since $J_{0}$ is linear and invariant to commutation, it follows that $J_{0}$ is an isomorphism between the Lie algebras $\mathfrak{g}_{1}$ and $\mathfrak{g}_{K}$.

Furthermore, let $\chi \in \mathfrak{g}_{1}$ and $\psi_{k} \in \mathfrak{g}_{k}, k \leq K-1$; then

$$
\begin{aligned}
& \chi \oplus 0 \oplus \cdots \oplus 0 \oplus J_{0}(\chi) \in \mathbb{L}_{\mathcal{A}, \mathcal{B}} \\
& \psi_{1} \oplus \cdots \oplus \psi_{K-1} \oplus J\left(\psi_{1}, \ldots, \psi_{K-1}\right) \in \mathbb{L}_{\mathcal{A}, \mathcal{B}} .
\end{aligned}
$$

Computing the commutator, we obtain

$$
\left[\chi, \psi_{1}\right] \oplus 0 \oplus \cdots \oplus 0 \oplus\left[J_{0}(\chi), J\left(\psi_{1}, \ldots, \psi_{K-1}\right)\right] \in \mathbb{L}_{\mathcal{A}, \mathcal{B}},
$$

and the definition of $J$ and $J_{0}$ imply that $J_{0}\left(\left[\chi, \psi_{1}\right]\right)=\left[J_{0}(\chi), J\left(\psi_{1}, \ldots, \psi_{K-1}\right)\right]$ Since $J_{0}$ is a morphism of Lie algebras, we obtain $\left[J_{0}(\chi), J_{0}\left(\psi_{1}\right)\right]=\left[J_{0}(\chi), J\left(\psi_{1}, \ldots, \psi_{K-1}\right)\right]$. This can be written $\left[J_{0}(\chi), J\left(0, \psi_{2}, \ldots, \psi_{K-1}\right)\right]=0$. But $J_{0}$ is surjective therefore $\left[Z, J\left(0, \psi_{2}, \ldots, \psi_{K-1}\right)\right]=0$ for all $Z \in \mathfrak{g}_{1}$. The Lie algebra $\mathfrak{g}_{1}$ is (simple thus) semi-simple which means that the above equation implies $J\left(0, \psi_{2}, \ldots, \psi_{K-1}\right)=0$ for any $\psi_{2} \in \mathfrak{g}_{2}, \ldots, \psi_{K-1} \in \mathfrak{g}_{K-1}$. In particular, $J_{0}\left(A_{1}\right)=J\left(A_{1}, 0, \ldots, 0\right)=J\left(A_{1}, \ldots, A_{K-1}\right)=A_{K}$ and similarly $J_{0}\left(B_{1}\right)=B_{K}$, Q.E.D.

Remark 1. 1. When $\operatorname{dim}_{\mathbb{R}} g_{k}$ are all different the individual controllability implies ensemble controllability. From this point of view the situation $\mathfrak{g}_{k}=\mathfrak{g}$ (for all $k$ ) is the most difficult.

2. When $\mathfrak{g}_{k}=\mathfrak{g}$ (for all $k$ ) one can exploit the structure of $\mathfrak{g}$. For the remarkable example $\mathfrak{g}=s u(N)$, we know that any automorphism is either $\chi \mapsto Y \chi Y^{-1}$ or $\chi \mapsto Y \bar{\chi} Y^{-1}$ for some $Y \in S U(N)(\bar{\chi}$ denotes the element-wise complex conjugation). In any such situation, it is enough to know if some $Y$ exists such that $A_{\ell}=Y A_{k} Y^{-1}, B_{\ell}=Y B_{k} Y^{-1}$ or $A_{\ell}=Y \overline{A_{k}} Y^{-1}, B_{\ell}=Y \overline{B_{k}} Y^{-1}$.
3. The result is not true for semi-simple Lie algebras. In this case the non-controllability is equivalent to the existence of an isomorphism between an ideal of some $\mathfrak{g}_{k}$ and an ideal of some $\mathfrak{g}_{\ell}$.

4. The result extends easily to the situation of several controls.

Using the previous results we can now treat the situation where the control seen by the $k$-th system is $u(t)+\alpha_{k}$ and not $u(t) \alpha_{k}$ as in [22].

Theorem 4. Consider $K \geq 1$ and $\alpha_{k} \in \mathbb{R}, k=1, . ., K$. The collection of systems (미) is simultaneously controllable if and only if $\mathbb{L}_{A, B}=\mathfrak{g}$ and $\alpha_{k} \neq \alpha_{\ell}$ for any $k \neq \ell$. In this case, there exists $T_{A, B, \alpha_{1}, \cdots, \alpha_{K}}>0$ such that the system is controllable in any time $t \geq T_{A, B, \alpha_{1}, \cdots, \alpha_{K}}$ with controls $u$ such that $|u(s)| \leq 1, \forall s \in[0, t]$.

Proof. In the view of the Theorem $\square$ the condition $\mathbb{L}_{A, B}=\mathfrak{g}$ is necessary. Of course $\alpha_{k} \neq \alpha_{\ell}$ for any $k \neq \ell$ is also required otherwise the same system with the same control appears twice in the list.

To assess controllability of (ㅁ), we consider it as a control system on $\oplus_{k=1}^{K} G$ given by matrices $\mathcal{A}=(A+$ $\left.\alpha_{1} B\right) \oplus \cdots \oplus\left(A+\alpha_{K} B\right)$ and $\mathcal{B}=B \oplus \cdots \oplus B$. Simultaneous controllability is equivalent to proving that $\mathbb{L}_{\mathcal{A}, \mathcal{B}}$ is isomorphic with $\oplus_{k=1}^{K}$ g.

Suppose that this is not the case; then by Lemma B, there exist $k \neq \ell$ and an automorphism $f: \mathfrak{g} \rightarrow \mathfrak{g}$ such that $f\left(A+\alpha_{k} B\right)=A+\alpha_{\ell} B$ and $f(B)=B$. Denote $\beta=\alpha_{\ell}-\alpha_{k} \neq 0$ then $f(A)=A+\beta B$.

Denote by $A u t(\mathfrak{g})$ the group of automorphisms of $\mathfrak{g}$. We recall that $A u t(\mathfrak{g})$ is compact (see [38, 39, 41] or any classical Lie theory textbook). Indeed, from the definition of the Killing form $K_{\mathfrak{g}}(\chi, \psi)=\operatorname{Tr}_{\mathfrak{g}}([\chi, \cdot] \circ[\psi, \cdot])$ it follows that any automorphism $h \in \operatorname{Aut}(\mathfrak{g})$ is such that $K_{\mathfrak{g}}(h(\chi), h(\psi))=K_{\mathfrak{g}}(\chi, \psi)$. Since $\mathfrak{g}$ is connected, compact, simple (thus semi-simple) the Killing form is negative definite and thus $\operatorname{Aut}(\mathfrak{g})$ is isomophic to a closed Lie subgroup of the orthogonal group of $O\left(\operatorname{dim}_{\mathbb{R}} \mathrm{g} ; \mathbb{R}\right)$ therefore $A u t(\mathfrak{g})$ is compact.

On the other hand

$$
\begin{aligned}
& A=f(A)-\beta B=-\beta B+f(-\beta B+f(A)) \\
& =-2 \beta B+f(f(A))=\ldots=-m \beta B+f^{m}(A) .
\end{aligned}
$$

Here, the automorphism $f^{m}$ is the $m$-th power of the automorphism $f$. We obtain thus:

$$
B=-\frac{A-f^{m}(A)}{\beta m}, \forall m=1,2, \ldots
$$

All $f^{m}$ live in the compact set $A u t(\mathfrak{g})$ thus the sequence $f^{m}(A)$ is bounded and, passing to the limit in the equation (D), we obtain $B=0$ which is impossible, Q.E.D. 
Corollary 5. Consider the bilinear system in equation (В)), where $G_{k}=G$ and $A_{k}=\epsilon_{k} A, B_{k}=B, \epsilon_{k} \in \mathbb{R}$, $k=1, . ., K$. Suppose $\left|\epsilon_{k}\right| \neq\left|\epsilon_{\ell}\right|$ for any $k \neq \ell$ and $\mathbb{L}_{A, B}=\mathfrak{g}$; then the collection of systems (B) is ensemble controllable.

Proof. We use the same arguments as in the previous result. Let $f$ be an automorphism of $\mathfrak{g}$, with $f\left(\epsilon_{k} A\right)=$ $\epsilon_{\ell} A$. Since $\left|\epsilon_{k}\right| \neq\left|\epsilon_{\ell}\right|$ there exists $\lambda \in \mathbb{R},|\lambda| \neq 1$ such that $f(A)=\lambda A$. Suppose for instance $|\lambda|>1$ (otherwise use $\left.f^{-1}\right)$. Then $f^{m}(A)=\lambda^{m} A$ and the contradiction is obtained because all $f^{m}$ live in a compact set.

Remark 2. 1. The Theorem 4 is not true for semisimple Lie groups. For instance let $\chi, \psi \in \operatorname{su}(N)$ such that $\mathbb{L}_{\chi, \psi}=\operatorname{su}(N)$ and $A=\chi \oplus(\chi+\psi) \in$ $s u(N) \oplus s u(N), B=\psi \oplus \psi \in \operatorname{su}(N) \oplus s u(N), \alpha_{1}=$ $0, \alpha_{2}=1$. The result above implies that $(A, B)$ is controllable as a system on $S U(N) \oplus S U(N)$. However the matrices $\mathcal{A}$ and $\mathcal{B}$ corresponding to the collection of systems $A+\left(u(t)+\alpha_{k}\right) B$ are $\mathcal{A}=$ $\chi \oplus(\chi+\psi) \oplus(\chi+\psi) \oplus(\chi+2 \psi)$ and respectively $\mathcal{B}=\psi \oplus \psi \oplus \psi \oplus \psi$. We note that the second and the third component are identical thus the system is not controllable.

2. The assumptions of the Corrolary 5 are weaker than those present in the literature. In [30], the same conclusion is obtained under the additional hypothesis that the transitions of $i A$ are nondegenerate (i.e., $A$ is "strongly regular" in the terminology of the Definition 2 in [30]). Recall that a matrix $\psi$ with eigenvalues $\lambda_{1}^{\psi}, \ldots, \lambda_{N}^{\psi}$ has no degenerate transitions if $\lambda_{a}^{\psi}-\lambda_{b}^{\psi} \neq \lambda_{i}^{\psi}-\lambda_{j}^{\psi}$ for all $(a, b) \neq(i, j)$.

3. Additional results can be easily constructed along the same lines, for instance for cases where the perturbation is not additive but on the form $\alpha_{k} u(t)+\beta_{k}$.

4. Having proved the results above for the bilinear setting, it is interesting to compare with the analogous result in the linear case. For this we consider the following linear systems:

$$
\begin{aligned}
& \frac{d}{d t} x_{1}=A x_{1}+B u(t), x_{1}(0)=0 \\
& \frac{d}{d t} x_{2}=A x_{2}+B[u(t)+\alpha], x_{2}(0)=0 .
\end{aligned}
$$

The dynamics of $x_{2}(t)-x_{1}(t)$ is not influenced by the control since $\frac{d}{d t}\left(x_{2}-x_{1}\right)=A\left(x_{2}-x_{1}\right)+$ $B \alpha, x_{2}(0)-x_{1}(0)=0$. Hence this collection of systems is never simultaneously controllable.
The Theorem 4 can be extended to the situation when the perturbations of the control depend on time. We will require however that the perturbations be constant on a common, long enough, time interval.

Corollary 6. Consider the collection of control systems with control $u(t)$.

$$
\frac{d Y_{k}(t)}{d t}=\left\{A+\left(u(t)+\beta_{k}(t)\right) B\right\} Y_{k}(t), Y_{k}(0)=Y_{k, 0} \in G .
$$

Suppose that $\mathbb{L}_{A, B}=\mathfrak{g}$ and there exists $0<t_{1}<t_{2}<\infty$ such that $\beta_{k}(t)=\alpha_{k}$ (constant) $\forall t \in\left[t_{1}, t_{2}\right]$ and $\alpha_{k} \neq$ $\alpha_{\ell}$ for $k \neq \ell$. Then there exists $T_{A, B, \alpha_{1}, \cdots, \alpha_{K}}$ such that if $t_{2}-t_{1} \geq T_{A, B, \alpha_{1}, \cdots, \alpha_{K}}$ the collection of systems (8) $i s$ simultaneously controllable at any time $T \geq t_{2}$.

Proof. Let $V_{k}$ be given targets for the systems (8) at time $T \geq t_{2}$. Define $u(t)$ to be zero on $\left[0, t_{1}\right] \cup\left[t_{2}, T\right]$ and $V_{k}^{-}=Y_{k}^{-}\left(t_{1}\right)$ where $Y_{k}^{-}(t)$ is the solution of $\frac{d Y_{k}^{-}(t)}{d t}=$ $\left(A+\beta_{k}(t) B\right) Y_{k}^{-}(t), Y_{k}^{-}(0)=Y_{k, 0}$ and $V_{k}^{+}=Y_{k}^{+}(T)$ where $Y_{k}^{+}(t)$ satisfies $\frac{d Y_{k}^{+}(t)}{d t}=\left(A+\beta_{k}(t) B\right) Y_{k}^{+}(t), Y_{k}^{+}\left(t_{2}\right)=I d$. Set targets $W_{k}=\left(V_{k}^{+}\right)^{-1} V_{k}\left(V_{k}^{-}\right)^{-1}$ for the system (Z) on $\left[0, t_{2}-t_{1}\right]$ and initial states $X_{k}(0)=I d$ and let $\tilde{u}(t)$ be the control that drives $X_{k}$ from $X_{k}(0)=I d$ to $X_{k}\left(t_{2}-t_{1}\right)=$ $W_{k}, \forall k=1, \cdots, K$. Then the control $u(s)$ with $u(s)=0$, for $s \in\left[0, t_{1}[\cup] t_{2}, T\right]$ and $u(s)=\tilde{u}\left(s-t_{1}\right)$, for $s \in\left[t_{1}, t_{2}\right]$ is such that $Y_{k}(T)=V_{k}^{+} W_{k} V_{k}^{-}=V_{k}$, Q.E.D.

\subsection{Further results on related models}

Note that the model in Equation (II) implies that the perturbation $\alpha_{k}$ is present even when the control $u(t)$ is null. In practice, it may sometimes be possible to eliminate the perturbations when the control field is not used and in this situation the controller can switch between a free, unperturbed dynamics and a controlled, perturbed one. This circumstance is modeled as

$$
\frac{d Z_{k}(t)}{d t}=A Z_{k}(t)+\left[u(t)+\alpha_{k}\right] \xi(t) B Z_{k}(t), Z_{k}(0) \in G,
$$

where the controls are $u(t)$ and $\xi(t)$, but $\xi(t) \in\{0,1\} \forall t \geq$ 0 ( $\xi$ being a measurable function). We obtain the following

Corollary 7. The system (四) is simultaneously controllable if and only if $\mathbb{L}_{A, B}=\mathfrak{g}$ and $\alpha_{k} \neq \alpha_{\ell}$ for any $k \neq \ell$.

Proof. Let $\xi(t)=1$ and apply the Theorem 4 . Of course $\mathbb{L}_{A, B}=\mathfrak{g}$ and $\alpha_{k} \neq \alpha_{\ell}$ for any $k \neq \ell$ are necessary conditions for controllability, which proves the reverse implication, Q.E.D. 
Remark 3. For the situation (Q9) a result analogous to Corollary 6 can be proved. We leave the proof as an exercise to the reader. In addition, both results remain true when $\xi$ is piecewise constant (with a discrete set of discontinuities).

\section{Application to the control of a quantum system}

Consider now a quantum bilinear system (cf. [5, 9, [14, 42]):

$$
\begin{aligned}
i \frac{d}{d t} \psi & =\left[H_{0}+u(t) \mu\right] \psi(t), \\
H_{0} & =\left(\begin{array}{ccccc}
1.0 & 0 & 0 & 0 & 0 \\
0 & 1.2 & 0 & 0 & 0 \\
0 & 0 & 1.3 & 0 & 0 \\
0 & 0 & 0 & 2.0 & 0 \\
0 & 0 & 0 & 0 & 2.15
\end{array}\right), \\
\mu & =\left(\begin{array}{ccccc}
0 & 0 & 0 & 1 & 1 \\
0 & 0 & 0 & 1 & 1 \\
0 & 0 & 0 & 1 & 1 \\
1 & 1 & 1 & 0 & 0 \\
1 & 1 & 1 & 0 & 0
\end{array}\right),
\end{aligned}
$$

controlled by the control $u(t)$ and with $\psi(0)=(1 / \sqrt{2}, 0,0,1 / \sqrt{2}, 0)^{T}$ and target $\psi_{T}=(0,1 / \sqrt{2}, 0,0,1 / \sqrt{2})^{T}$. This system has been extensively used (see the references above) as a benchmark for testing the controllability of bilinear quantum finite-dimensional systems: controllability criterions, search algorithms to find the controls etc. It has no degenerate transitions but a bi-partite connectivity graph structure: the set of eigenstates 1 to 3 are not directly connected, same for 4 and 5. Thus transferring population from eigenstate 1 to 2 requires a second-order excitation using eigenstates 4 or 5 as intermediary. Define $B=\mu / i$ and, for simplicity, $A=\left[H_{0}-0.2 \operatorname{Tr}\left(H_{0}\right) . I d\right] / i$ such that both $A$ and $B$ belong to $s u(5)$. Using the tool in [43], we obtain $\operatorname{dim}_{\mathbb{R}} \mathbb{L}_{A, B}=24=\operatorname{dim}_{\mathbb{R}} \operatorname{su}(5)$ thus $\mathbb{L}_{A, B}=\operatorname{su}(5)$. Consider the perturbations $\alpha_{1}=-0.1, \alpha_{2}=0, \alpha_{3}=0.1$. Therefore Theorem $\mathbf{4}$, Corollary $\mathbf{6}$ and Corrolary $\square$ of the previous section apply. Since $S U(5)$ is transitive on the unit sphere of $\mathbb{C}^{5}$ (cf. [7]) there exists $U_{T} \in S U(5)$ such that $U_{T} \psi_{0}=\psi_{T}$ and by the Theorem $⿴$ there exists a time $T$ and a control $u:[0, T] \rightarrow \mathbb{R}$ such that $u(t), u(t)-0.1$ and $u(t)+0.1$ all drive $I d$ to $U_{T}$ in

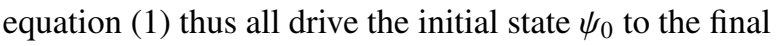
state $\psi_{T}$ in equation (10). We searched numerically the control $u(t)$ using a so-called monotonic procedure, see
[44-49] for details. For $T=500$, we obtain the control presented in Figure $\square$. The quality of the control, i.e. the quantity $\frac{|\langle\psi(T), \psi(0)\rangle|}{\|\psi(0)\|}$ is over $99 \%$ for all perturbations $\alpha_{k}, k=1,2,3$. We also tested different pairs of initial and target states $\left(\psi_{0}, \psi_{T}\right)$ and in all cases high quality controls were found.

\section{Extensions to an infinite set of perturbations}

We investigate in this section the circumstance when $K$ (the number of perturbations) is infinite. The controllability of a system consisting of an infinite collection of finite-dimensional systems has been analyzed for the situation of the Bloch equation $(G=S O(3))$ in [2527, 32]. To the best of our knowledge no general results are available for generic systems and values of $N$; moreover the counter-example in Theorem 4 in [27] warns that general results may be impossible to obtain.

We explore two questions: first we give an example that builds on the Maxwell-Bloch equation where a positive controllability result is expected; next we give a procedure for the numerical identification of approximate controls of a Bloch equation.

\subsection{An example of perturbed Maxwell-Bloch equation}

Let $\Omega$ a compact subset of $\mathbb{R}^{3}$ and recall the notation for the Pauli matrices:

$$
\sigma_{x}=\left(\begin{array}{ll}
0 & 1 \\
1 & 0
\end{array}\right), \sigma_{y}=\left(\begin{array}{cc}
0 & -i \\
i & 0
\end{array}\right), \sigma_{z}=\left(\begin{array}{cc}
1 & 0 \\
0 & -1
\end{array}\right) .
$$

Consider the Maxwell-Bloch equation with two controls:

$$
\begin{aligned}
& i \frac{d X(t, \omega, \alpha, \beta)}{d t}=\left\{\omega \sigma_{z}+[u(t)+\alpha] \sigma_{x}+\right. \\
& \left.[v(t)+\beta] \sigma_{y}\right\} X(t, \omega, \alpha, \beta), \\
& X(0, \omega, \alpha, \beta)=I d,(\omega, \alpha, \beta) \in \Omega .
\end{aligned}
$$

Proposition 8. Let $f=\left(f_{x}, f_{y}, f_{z}\right): \Omega \rightarrow \mathbb{R}^{3}$ be a continuous function. Then, for any $\eta>0$ there exists a time $T_{\eta}>0$ and two controls $u_{\eta}, v_{\eta} \in L^{\infty}\left(\left[0, T_{\eta}\right]\right)$ such that for all $(\omega, \alpha, \beta) \in \Omega$ :

$$
\left\|X\left(T_{\eta}, \omega, \alpha, \beta\right)-e^{i\left(f_{x}(\omega, \alpha, \beta) \sigma_{x}+f_{y}(\omega, \alpha, \beta) \sigma_{y}+f_{z}(\omega, \alpha, \beta) \sigma_{z}\right)}\right\| \leq \eta .
$$

Proof. Although a rigorous proof of the controllability would require the tools in [32] and is beyond the scope of this work, we give below the arguments that indicate that this system is controllable. Consider the sequence of controls: start with $u=-(\pi / 2) \delta_{0}$ ( $\delta_{0}$ is the Dirac 


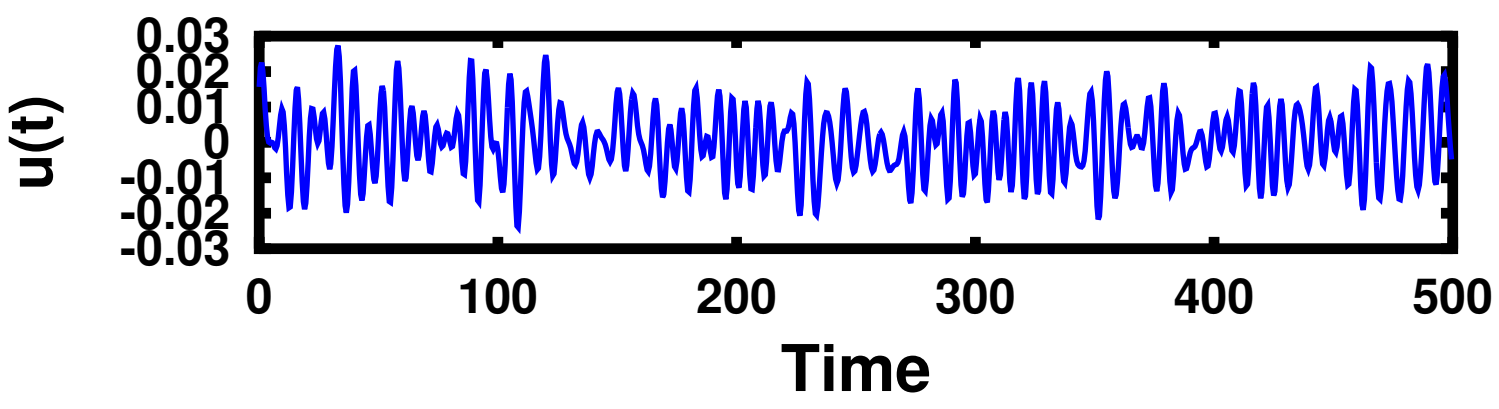

Figure 1: The control that drives $\psi_{0}$ to $\psi_{T}$ (cf. equation (피) ) irrespective of the perturbation $\alpha_{k} \in\{-0.1,0,0.1\}$. The quality of the control is over $99 \%$ for any perturbation. However the trajectories $\psi(t)$ corresponding to $u(t)-0.1, u(t)$ and $u(t)+0.1$ are all different.

mass at the origin), followed by free evolution during a unit of time and then $u=+(\pi / 2) \delta_{1}$. That is, choose $u=$ $-(\pi / 2) \delta_{0}+(\pi / 2) \delta_{1}, v=0$. This results in the evolution

$$
e^{-i(\pi / 2) \sigma_{x}} e^{-i\left(\omega \sigma_{z}+\alpha \sigma_{x}+\beta \sigma_{y}\right)} e^{i(\pi / 2) \sigma_{x}}=e^{-i\left(-\omega \sigma_{z}+\alpha \sigma_{x}-\beta \sigma_{y}\right)} .
$$

Thus the propagator associated with $-\omega \sigma_{z}+\alpha \sigma_{x}-\beta \sigma_{y}$ can be synthesized. A similar computation (now using the control $v$ ) allows to construct $-\omega \sigma_{z}-\alpha \sigma_{x}+\beta \sigma_{y}$. Using now infinitesimal times and the formula $e^{U+V}=$ $\lim _{n \rightarrow \infty}\left(e^{U / n} e^{V / n}\right)^{n}$, we have thus at our disposal all propagators $e^{ \pm i \omega \sigma_{z}}, e^{ \pm i \alpha \sigma_{x}}, e^{ \pm i \beta \sigma_{y}}$. Recall that we also have $e^{ \pm i \sigma_{x}}, e^{ \pm i \sigma_{y}}$.

From now on, the argument is similar to that in [27]: the formula

$$
\lim _{n \rightarrow \infty}\left\{e^{-\chi / n} e^{-\psi / n} e^{\chi / n} e^{\psi / n}\right\}^{n^{2}}=e^{[\chi, \psi]},
$$

allows to use commutators of, for instance, $\pm i \omega \sigma_{z}$ and $\pm i \sigma_{x}$ which produce $\pm i \omega \sigma_{y}$ and then commutators $\pm i \omega \sigma_{z}$ and $\pm i \omega \sigma_{y}$, which produce $\pm i \omega^{2} \sigma_{x}$; all other polynomials of $\omega$ can be obtained as multiplicative factors in front of $\omega_{z}$. Similar arguments allow to further obtain all possible polynomials of three variables $\omega, \alpha, \beta$. Therefore we obtain approximate controllability of the system to any (smooth) target with $L^{\infty}$ controls.

Remark 4. 1. The result extends obviously to the Bloch equation (set on $S O(3)$, see next section).

2. Not all situations have favorable outcomes. For instance, using same arguments as in Remark page 030302-2 of [26], it is possible to show that for the controlled Hamiltonian $\sigma_{z}+\alpha \sigma_{y}+u(t) \sigma_{x}$ the unknown perturbation $\alpha \in$ ] $\alpha_{*}, \alpha^{*}$ [ cannot always be compensated. Indeed, the attainable propagators are of the form

$$
\exp \left\{i f_{1}\left(\alpha^{2}\right)\left(\sigma_{y}-\alpha \sigma_{z}\right)+i f_{2}\left(\alpha^{2}\right)\left(\sigma_{z}+\alpha \sigma_{y}\right)+i f_{3}\left(\alpha^{2}\right) \sigma_{x}\right\}
$$

where $f_{1}, f_{2}$ and $f_{3}$ are arbitrary functions. Thus when for instance $\Omega$ is symmetric with respect to $\alpha$ the functions $f_{1}, f_{2}, f_{3}$ are odd functions which is a restriction for controllability.

\subsection{Convergence of the controls for a discrete set of perturbations}

We investigate here a numerical algorithm to find the control when the set of perturbations can be a whole (possibly unbounded) closed interval $I_{\alpha} \subset \mathbb{R}$. Suppose $A, B \in \mathfrak{g}$ are such that $\mathbb{L}_{A, B}=\mathfrak{g}$ and let us denote by $X(t, \alpha, u)$ the solution of $\frac{d X(t)}{d t}=(A+(u(t)+\alpha) B) X$ at time $t$ starting from $X(0)=I d$.

Consider also a continuous cost function to be minimized $F: I_{\alpha} \times G \rightarrow \mathbb{R}_{+}$and to fix notations suppose that for any $\alpha \in I_{\alpha}$ there exists some $Z_{\alpha} \in G$ with $F\left(\alpha, Z_{\alpha}\right)=0$. One interesting example of such function is the distance $F(\alpha, Z)=\|Z-Y(\alpha)\|$ to some predefined target $Y(\alpha)$ continuous with respect to $\alpha \in I_{\alpha}$. Of course $Y$ can be in particular constant with respect to $\alpha$. Consider a sequence of divisions $\mathcal{T}_{\ell} \subset I_{\alpha}$ : $\alpha_{1}^{\ell}<\alpha_{1}^{\ell}<\ldots<\alpha_{K_{\ell}}^{\ell}$ of the interval $I_{\alpha}$ such that $\left|\mathcal{T}_{\ell}\right|:=\max _{j=2, K_{\ell}}\left|\alpha_{j}^{\ell}-\alpha_{j-1}^{\ell}\right|$ tends to 0 when $\ell$ tends to $\infty$. Fix also a tolerance $\eta \geq 0$. Using the results of the previous sections there exists a time $T_{\ell}$ and a control $u_{\ell}$ such that $F\left(\alpha_{j}^{\ell}, X\left(T_{\ell}, \alpha_{j}^{\ell}, u_{\ell}\right)\right) \leq \eta$ for all $j=1, \ldots, K_{\ell}$. In this section, we give a sufficient result that ensures the existence of a control $u$ that minimizes the cost $F$ for the whole interval of perturbations $I_{\alpha}$ up to the tolerance $\eta$.

Proposition 9. Suppose that the sequence $T_{\ell}$ is not converging to infinity and $\left\|u_{\ell}\right\|_{L^{r}\left(\left[0, T_{\ell}\right]\right)}$ are bounded by a common constant for some $1<r<\infty$. Then there 
exists $T>0$ and $u \in L^{r}([0, T])$ (independent of $\alpha$ ) such that $F(\alpha, X(T, \alpha, u)) \leq \eta$, for all $\alpha \in I_{\alpha}$.

Proof. Since $T_{\ell}$ does not converge to $\infty$ it has a subsequence converging to some $T \in \mathbb{R}$. Denote again by $T_{\ell}$ this subsequence; we can moreover consider that all $T_{\ell}$ are either greater or smaller than $T$, let us say $T_{\ell} \leq T$ for all $\ell$. Extend the domain of definition of $u_{\ell}$ on $[0, T]$ with $u_{\ell}=0$ on $\left[T_{\ell}, T\right]$; this will not change its $L^{r}$ norm. Up to extracting another subsequence, there exists $u \in L^{r}([0, T])$ such that $u_{\ell}$ converges weakly in $L^{1}([0, T])$ to $u$. Let us prove that $u$ satisfies the required conditions. Fix $\alpha \in I_{\alpha}$. Since $\left|\mathcal{T}_{\ell}\right| \rightarrow 0$, there exists a sequence $\alpha_{k_{\ell}}^{\ell}$ such that $\alpha_{k_{\ell}}^{\ell} \rightarrow \alpha$ when $\ell \rightarrow \infty$. We write:

$$
\begin{aligned}
& \left\|X\left(T_{\ell}, \alpha_{k_{\ell}}^{\ell}, u_{\ell}\right)-X(T, \alpha, u)\right\| \\
& \leq\left\|X\left(T_{\ell}, \alpha_{k_{\ell}}^{\ell}, u_{\ell}\right)-e^{\left(T-T_{\ell}\right) A} X\left(T_{\ell}, \alpha_{k_{\ell}}^{\ell}, u_{\ell}\right)\right\| \\
& +\left\|e^{\left(T-T_{\ell}\right) A} X\left(T_{\ell}, \alpha_{k_{\ell}}^{\ell}, u_{\ell}\right)-X(T, \alpha, u)\right\| .
\end{aligned}
$$

The term $\left\|X\left(T_{\ell}, \alpha_{k_{\ell}}^{\ell}, u_{\ell}\right)-e^{\left(T-T_{\ell}\right) A} X\left(T_{\ell}, \alpha_{k_{\ell}}^{\ell}, u_{\ell}\right)\right\|$ is bounded by $C\left\|I d-e^{\left(T-T_{\ell}\right) A}\right\|$ for some constant $C>0$ and thus converges to 0 . The last term can be written as:

$$
\begin{aligned}
& \left\|e^{\left(T-T_{\ell}\right) A} X\left(T_{\ell}, \alpha_{k_{\ell}}^{\ell}, u_{\ell}\right)-X(T, \alpha, u)\right\| \\
& =\left\|e^{\left(T-T_{\ell}\right) A} X\left(T_{\ell}, 0, \alpha_{k_{\ell}}^{\ell}+u_{\ell}\right)-X(T, 0, \alpha+u)\right\| \\
& =\| X\left(T, 0, \alpha_{k_{\ell}}^{\ell}+u_{\ell}+1_{\left[T_{\ell}, T\right]} \cdot\left(-\alpha_{k_{\ell}}^{\ell}\right)\right) \\
& -X(T, 0, \alpha+u) \| .
\end{aligned}
$$

Since $T_{\ell} \rightarrow T$, it follows that $\alpha_{k_{\ell}}^{\ell}+u_{\ell}+1_{\left[T_{\ell}, T\right]} \cdot\left(-\alpha_{k_{\ell}}^{\ell}\right)$ converges weakly in $L^{1}([0, T])$ to $\alpha+u$. From Theorem 3.6 of [50] (see also the Aubin-Lions lemma [5]]), the weak convergence of $\alpha_{k_{\ell}}^{\ell}+u_{\ell}+1_{\left[T_{\ell}, T\right]} \cdot\left(-\alpha_{k_{\ell}}^{\ell}\right)$ to $\alpha+u$ ensures that $\lim _{\ell \rightarrow \infty} X\left(T, 0, \alpha_{k_{\ell}}^{\ell}+u_{\ell}+1_{\left[T_{\ell}, T\right]} \cdot\left(-\alpha_{k_{\ell}}^{\ell}\right)\right)=$ $X(T, 0, \alpha+u)$. Combining all estimations, we obtain $\lim _{\ell \rightarrow \infty} X\left(T_{\ell}, \alpha_{k_{\ell}}^{\ell}, u_{\ell}\right)=X(T, \alpha, u)$ thus

$$
F(\alpha, X(T, \alpha, u))=\lim _{\ell \rightarrow \infty} F\left(\alpha_{k_{\ell}}^{\ell}, X\left(T_{\ell}, \alpha_{k_{\ell}}^{\ell}, u_{\ell}\right)\right) \leq \eta,
$$

and the conclusion follows.

Remark 5. The Proposition is not a controllability result but can be used numerically to find the control when controllability holds true.

In particular the situation $\eta=0$ corresponds to exact controllability; however the results in [32] show that approximate controllability is more likely to hold and the controls will be in $L_{l o c}^{\infty}$, thus in all $L^{r}([0, t])$

As a numerical illustration we consider the Bloch equation (which is a perturbation of the system in [32] for $\left.\omega=\omega_{0}\right)$ :

$\frac{d}{d t}\left(\begin{array}{l}M_{x} \\ M_{y} \\ M_{z}\end{array}\right)=\left(\begin{array}{ccc}0 & -(u(t)+\alpha) & 0 \\ u(t)+\alpha & 0 & -\omega_{0} \\ 0 & \omega_{0} & 0\end{array}\right)\left(\begin{array}{l}M_{x} \\ M_{y} \\ M_{z}\end{array}\right),\left(\begin{array}{l}M_{x}(0) \\ M_{y}(0) \\ M_{z}(0)\end{array}\right)=$ where $u(t)$ is the control. The system can be put into the framework of Proposition $Q$ by considering $G=S O(3)$, $A=\omega_{0}\left(\begin{array}{ccc}0 & 0 & 0 \\ 0 & 0 & -1 \\ 0 & 1 & 0\end{array}\right), B=\left(\begin{array}{ccc}0 & -1 & 0 \\ 1 & 0 & 0 \\ 0 & 0 & 0\end{array}\right)$.

Let $M_{f}$ be some target state. The goal to steer $M_{0}$ to $M_{f}$ at time $T$ can be rephrased as minimizing, with respect to $u, F(\alpha, X(T, \alpha, u))$ where $F(\alpha, Z)=$ $\left\|Z M_{0}-M_{f}\right\|$. The tolerance $\eta$ is set to $5 \%$. We take $M_{0}=(1,0,0)^{T}$ and $M_{f}=(0,0,1)^{T}$. The perturbation $\alpha$ takes all values in the interval $I_{\alpha}=\left[-\alpha_{\text {max }}, \alpha_{\text {max }}\right]$; the divisions $\mathcal{T}_{\ell}$ use a Tchebytchev-type grid containing the points $\alpha_{k}^{\ell}=\alpha_{\text {max }} \cos (k \pi / \ell)$ with $k=0, \cdots, K_{\ell}=\ell$. We consider the values of the parameters $\omega_{0}=50$, $T=1000, \alpha_{\max }=0.5$ For the numerical resolution of the evolution equation in $X(t, \alpha, u)$ we use a CrankNicholson time-discretization scheme, with $10^{3}$ time steps in $[0, T]$. To compute the optimal controls $u_{\ell}$ we apply again the monotonic procedure, see Section 4 .

In order to check the assumptions of Proposition $Q$ we set $r=2$ and verify that the $\left\|u_{\ell}\right\|_{L^{2}}$ remain bounded when $\ell$ increases. The norms $\left\|u_{\ell}\right\|_{L^{2}}$ are presented in Figure $\square$ and are uniformly bounded with respect to $\ell$. The quality of these controls is evaluated with

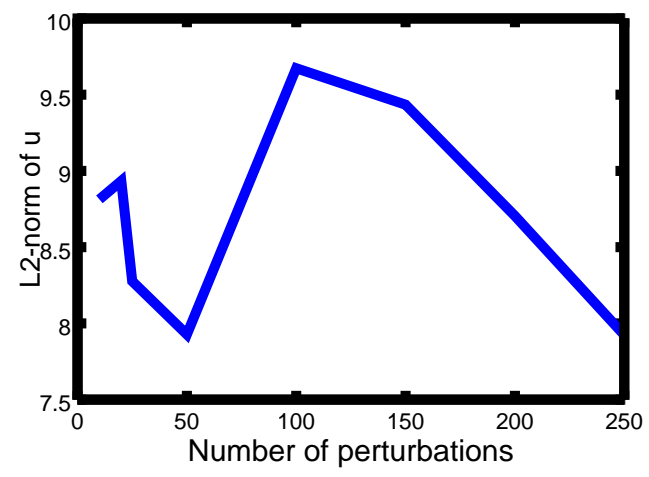

Figure 2: Norms of $\left\|u_{\ell}\right\|_{L^{2}}$ for $\ell=5,10,25,50,100,150,200,250$.

$F\left(\alpha, X\left(T, \alpha, u_{\ell}\right)\right)$, which is in all cases lower than $\eta$. In Figure B, $F\left(\alpha, X\left(T, \alpha, u_{\ell}\right)\right)$ is plotted as a function of $\alpha$ for the control field $u_{250}$. We observe a very accurate control in the whole interval $\alpha \in I_{\alpha}$ (but the quality decays outside this interval).

\section{Conclusion and perspectives}

Necessary and sufficient conditions have been derived for the ensemble controllability of a finite collecMijon of bilinear systems on a connected, compact, simple Lie group. The result was applied to the case where 


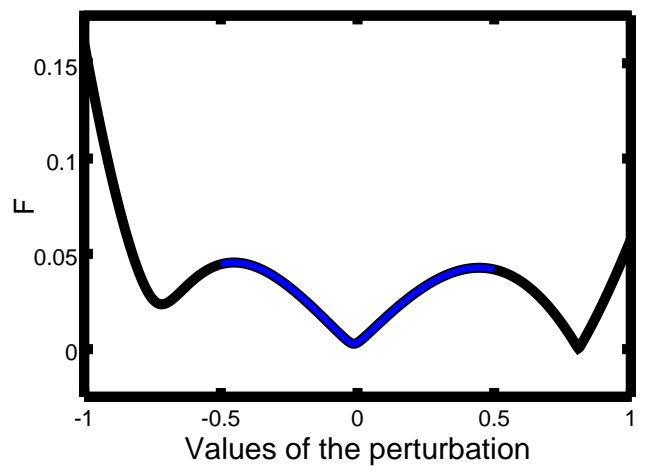

Figure 3: Values of $F\left(\alpha, X\left(T, \alpha, u_{\ell}\right)\right)$ for $\alpha \in[-1,1]$. The optimal control $u_{250}$ is applied. As expected the quality is within the tolerance $\eta$ in the interval $I_{\alpha}$ but not outside it.

the control is submitted to a finite collection of constant or partially constant perturbations. The result extends to ensemble controllability and generalizes several works from the literature. Additional arguments have been presented when the number of possible perturbations is infinite.

This work studied the controllability for possibly large final times. A related question is whether small time local controllability (called STLC) is also true. A further question is whether the result extends to more general, time dependent, perturbations.

\section{Acknowledgments}

MB acknowledges support from the "Institut Français de Tunisie" (IFT). JS \& GT acknowledge support from the Agence Nationale de la Recherche (ANR), Projet Blanc EMAQS number ANR-2011BS01-017-01. The authors thank C. Lefter ("Al. I. Cuza University" Iaşi, Romania) for helpful discussions concerning this work and for proposing the model in Section 3.3.

\section{References}

[1] E.-D. Sontag, Mathematical control theory: deterministic finite dimensional systems, 2nd Edition, Springer, 1998.

[2] H.-K. Khalil, Nonlinear systems, Macmillan, 1996.

[3] H. Nijmeiher, A. van der Schaft, Nonlinear dynamical control systems, Springer, 1990.

[4] F. S. Leite, P.-E. Crouch, Controllability on classical Lie groups, Mathematics of Control, Signals, and Systems 1 (1988) 31-42.

[5] V. Ramakrishna, M. Salapaka, M. Dahleh, H. Rabitz, A. Peirce, Controllability of molecular-systems, Physical Review A 51 (1995) 960-966.

[6] D. D'Alessandro, Small time controllability of systems on compact Lie groups and spin angular momentum, J. Math. Phys. 42 (9) (2001) 4488-4496.
[7] F. Albertini, D. D'Alessandro, Notions of controllability for quantum mechanical systems, in: 40th IEEE Conference on Decision and Control, Vol. 2, Orlando, FL, 2001, pp. 1589-1594.

[8] C. Altafini, Controllability of quantum mechanical systems by root space decomposition of $\mathrm{su}(\mathrm{n})$, Journal of Mathematical Physics 43 (5) (2002) 2051-2062.

[9] G. Turinici, H. Rabitz, Wavefunction controllability in quantum systems, Journal of Physics A: Mathematical and Theoretical 36 (2003) 2565-2576.

[10] S. Rice, M. Zhao, Optical control of quantum dynamics, Wiley, 2000.

[11] G. Huang, T. Tarn, J. Clark, On the controllability of quantum mechanical systems, J. Math. Phys. 24 (1983) 2608-2618.

[12] R. Judson, K. Lehmann, H. Rabitz, W.-S. Warren, Optimal design of external fields for controlling molecular motionapplication to rotation, J. Molec. Structure 223 (1990) 425-456.

[13] G. Turinici, On the controllability of bilinear quantum systems, in: M. Defranceschi, C. L. Bris (Eds.), Mathematical models and methods for ab initio Quantum Chemistry, Lecture Notes in Chemistry vol. 74, Springer, 2000, pp. 75-92.

[14] S.-G. Schirmer, H. Fu, A.-I. Solomon, Complete controllability of quantum systems, Phys. Rev. A 63 (2001) 063410.

[15] G. Turinici, H. Rabitz, Quantum Wave Function Controllability, Chemical Physics 267 (1-3) (2001) 1-9.

[16] M.-D. Girardeau, M. Ina, S.-G. Schirmer, T. Gulsrud, Kinematical bounds on evolution and optimization of mixed quantum states, Phys. Rev. A 55 (1997) R1565-R1568.

[17] K. Beauchard, Local controllability of a 1-D Schrödinger equation, Journal de Mathématiques Pures et Appliquées 84 (7) (2005) 851-956. doi: $\{10.1016 / j$.matpur.2005.02.005\}.

[18] K. Beauchard, C. Laurent, Local controllability of 1D linear and nonlinear Schrödinger equations with bilinear control, Journal de Mathématiques Pures et Appliquées 94 (5) (2010) 520-554. doi:\{10.1016/j.matpur.2010.04.001\}.

[19] M.-D. Girardeau, S.-G. Schirmer, J.-V. Leahy, R.-M. Koch, Kinematical bounds on optimization of observables for quantum states, Phys. Rev. A 58 (1998) 2684-2689.

[20] G. Turinici, V. Ramakrishna, B. Li, H. Rabitz, Optimal Discrimination of Multiple Quantum Systems: Controllability Analysis, J. Phys. A: Mathematical and General 37 (1) (2003) 273-282.

[21] B. Li, G. Turinici, V. Ramakhrishna, H. Rabitz, Optimal Dynamic Discrimination of Similar Molecules Through Quantum Learning Control, Journal of Physical Chemistry B 106 (33) (2002) 8125-8131.

[22] G. Turinici, H. Rabitz, Optimally controlling the internal dynamics of a randomly oriented ensemble of molecules, Phys. Rev. A 70 (6) (2004) 063412. doi:10.1103/PhysRevA.70. 063412 .

[23] H. Rabitz, G. Turinici, Controlling quantum dynamics regardless of laser beam spatial profile and molecular orientation, Physical review A: Atomic, Molecular and Optical Physics 75 (4) (2007) 043409.

[24] S.-G. Schirmer, I.-C. Pullen, A.-I. Solomon, Controllability of multi-partite quantum systems and selective excitation of quantum dots, Journal of Optics B 7 (2005) S293-S299.

[25] J.-S. Li, N. Khaneja, Ensemble controllability of the Bloch equations, in: 45th IEEE Conference on Decision and Control, San Diego, CA, USA, 2006, pp. 13-15.

[26] J.-S. Li, N. Khaneja, Control of inhomogeneous quantum ensembles, Phys. Rev. A 73 (2006) 030302.

[27] J.-S. Li, N. Khaneja, Ensemble control of Bloch equations, IEEE Trans. Automat. Control 54 (3) (2009) 528-536. तoi: 10.1109/TAC. 2009.2012983.

URL http://dx.doi.org/10.1109/TAC.2009.2012983

[28] D. Sugny, A. Keller, O. Atabek, D. Daems, C. M. Dion, 
S. Guerin, H.-R. Jauslin, Control of mixed-state quantum systems by a train of short pulses, Physical Review A (Atomic, Molecular, and Optical Physics) 72 (3) (2005) 032704.

[29] T.-J. Tarn, J. Clark, D. Lucarelli, Controllability of quantum mechanical systems with continuous spectra, in: Decision and Control, 2000. Proceedings of the 39th IEEE Conference on, Vol. 1, 2000, pp. 943-948 vol.1. doi:10.1109/CDC. 2000.912894.

[30] C. Altafini, Controllability and simultaneous controllability of isospectral bilinear control systems on complex flag manifolds, Systems \& Control Letters 58 (2009) 213-216.

[31] K. Moore, H. Rabitz, Manipulating molecules, Nature Chemistry 4 (2012) 72-73.

[32] K. Beauchard, J.-M. Coron, P. Rouchon, Controllability issues tor continuous-spectrum systems and ensemble controllability of Bloch equations, Comm. Math. Phys. 296 (2) (2010) 525557. doi:10.1007/s00220-010-1008-9. URL http://dx.doi.org/10.1007/s00220-010-1008-9

[33] K. Khodjasteh, L. Viola, Dynamical quantum error correction of unitary operations with bounded controls, Phys. Rev. A 80 (2009) 032314. doi:10.1103/PhysRevA.80.032314.

URL http://link.aps.org/doi/10.1103/PhysRevA. 80.032314

[34] K. Khodjasteh, L. Viola, Dynamically error-corrected gates for universal quantum computation, Phys. Rev. Lett. 102 (2009) 080501. doi:10.1103/PhysRevLett.102.080501. URL http://link.aps.org/doi/10.1103/ PhysRevLett.102.080501

[35] A. M. Souza, G. A. Álvarez, D. Suter, Experımental protection of quantum gates against decoherence and control errors, Phys. Rev. A 86 (2012) 050301. doi:10.1103/PhysRevA.86.050301.

URL http://link.aps.org/doi/10.1103/PhysRevA. 86.050301

[36] D. Hocker, C. Brif, M. D. Grace, A. Donovan, T.-S. Ho, K. W. Moore Tibbetts, R. Wu, H. Rabitz, Characterization of control noise effects in optimal quantum unitary dynamics, ArXiv eprintsVersion 1. arXiv:1405.5950.

[37] I. R. Sola, H. Rabitz, The influence of laser field noise on controlled quantum dynamics, The Journal of Chemical Physics 120 (19) (2004) 9009-9016. doi:http://dx.doi.org/10.1063/1.1691803

URL http://scitation.aip.org/content/aip/ journal/jcp/120/19/10.1063/1.1691803

[38] S. Helgason, Differential geometry, Lie groups, and symmetric spaces, Vol. 34 of Graduate Studies in Mathematics, American Mathematical Society, Providence, RI, 2001, corrected reprint of the 1978 original.

[39] W. A. de Graaf, Lie algebras: theory and algorithms, Vol. 56 of North-Holland Mathematical Library, North-Holland Publishing Co., Amsterdam, 2000. doi:10.1016/S0924-6509(00)80040-9.

URL http://dx.doi.org/10.1016/S0924-6509(00) 80040-9

[40] V. Jurdjevic, H. J. Sussmann, Control systems on Lie groups, J. Differ. Equations 12 (1972) 313-329.

[41] V. S. Varadarajan, Lie groups, Lie algebras, and their representatıons, Vol. 102 of Graduate Texts in Mathematics, SpringerVerlag, New York, 1984, reprint of the 1974 edition. doi: 10.1007/978-1-4612-1126-6

URL http://dx.doi.org/10.1007/978-1-4612-1126-6

[42] S. H. Tersigni, P. Gaspard, S. A. Rice, On using shaped light pulses to control the selectivity of product formation in a chemical reaction: An application to a multiple level system, The Journal of Chemical Physics 93 (3) (1990) 1670-1680. doi:http://dx.doi.org/10.1063/1.459680
URL http://scitation.aip.org/content/aip/ journal/jcp/93/3/10.1063/1.459680

[43] Online controllability calculator. URL https://www.ceremade.dauphine.fr/ turinici/ index.php/fr/recherche/calculator.html

[44] M. Belhadj, J. Salomon, G. Turinici, A stable toolkit method in quantum control, Journal of Physics A: Mathematical and Theoretical 41 (36) (2008) 362001-362011.

[45] D. Tannor, V. Kazakov, V. Orlov, Control of photochemical branching: Novel procedures for finding optimal pulses and global upper bounds, in: Time Dependent Quantum Molecular Dynamics ed J Broeckhove and L Lathouwers, New York: Plenum, 1992, pp. 347-360.

[46] W. Zhu, H. Rabitz, A rapid monotonically convergent iteration algorithm for quantum optimal control over the expectation value of a positive definite operator, J. Chem. Phys. 109 (1998) 385-391.

[47] S. G. Schirmer, M. D. Girardeau, J. V. Leahy, Efficient algorithm for optimal control of mixed-state quantum systems, Phys. Rev. A 61 (1999) 012101. doi:10.1103/PhysRevA.61.012101.

[48] L. Baudouin, J. Salomon, Constructive solution of a bilinear optimal control problem for a Schrödinger equation, Systems \& Control Letters 57 (6) (2008) 453-464. doi:\{10.1016/j. sysconle.2007.11.002\}.

[49] Y. Maday, G. Turinici, New formulations of monotonically convergent quantum control algorithms, J. Chem. Phys. 118 (2003) 8191-8196.

[50] J. Ball, J. Marsden, M. Slemrod, Controllability tor distributed bilinear systems, SIAM Journal on Control and Optimization 20 (4) (1982) 575-597. arXiv:http://dx.doi.org/10. 1137/0320042, doi:10.1137/0320042.

URL http://dx.doi.org/10.1137/0320042

[51] J.-P. Aubin, Un théorème de compacité, C. R. Acad. Sci. Paris 256 (1963) 5042-5044. 